\title{
Kinetics of visible light photo-oxidation of Ge nanocrystals: Theory and in situ measurement
}

I.D. Sharp, Q. Xu, C.W. Yuan

Materials Sciences Division, Lawrence Berkeley National Laboratory, Berkeley, CA 94720 and

Department of Materials Science \& Engineering, University of California, Berkeley, CA 94720

J.W. Beeman and J.W. Ager III

Materials Sciences Division, Lawrence Berkeley National Laboratory, Berkeley, CA 94720

D.C. Chrzan and E.E. Haller

Materials Sciences Division, Lawrence Berkeley National Laboratory, Berkeley, CA 94720 and

Department of Materials Science \& Engineering, University of California, Berkeley, CA 94720

Photo-oxidation of Ge nanocrystals illuminated with visible laser light under ambient conditions was investigated. The photo-oxidation kinetics were monitored by in situ measurement of the crystalline Ge volume fraction by Raman spectroscopy. The effects of laser power and energy on the extent of oxidation were measured using both in situ and ex situ Raman scattering techniques. A mechanistic model in which the tunneling of photo-excited carriers to the oxide surface for electron activated molecular oxygen dissociation is proposed. This quantitative model successfully describes all experimental photo-oxidation observations using physical parameters.

PACS numbers:81.16.Pr, 81.07.-b, 78.30.-j, 82.50.Hp 
Since the early days of semiconductor research, oxidation phenomena have attracted considerable attention due to their fundamental impact on surface electrical properties and device processing [1]. Recently, semiconductor nanostructures have become the subject of intense research. Because these materials necessarily possess large surface to volume ratios, understanding and controlling oxidation phenomena can be critical. For the case of Ge nanocrystals, it was previously found that exposed nanocrystals are stable under air after formation of a very thin native oxide layer [2]. Here, we show that exposed Ge nanocrystals can be photo-oxidized when irradiated with visible light. In situ Raman spectroscopy was used to monitor the volume fraction of nanocrystals consumed by oxidation as a function of time under irradiation and a quantitative, mechanistic model is proposed to describe the oxidation kinetics.

In 1939, Mott first proposed a mechanism for the oxidation of metals in which the rate is limited by the tunneling of thermal electrons through the growing oxide to electronic states of adsorbed oxygen at the surface [3]. Close to fifty years later, visible light photo-oxidation of bulk Si at elevated temperatures was observed by Young, who qualitatively proposed an electron-active oxidation mechanism similar to Mott's original tunneling theory, with the adaptation that photo-excitation injects the electrons into the oxide [4]. Though the fundamental physics of this oxidation process have been previously proposed, we are unaware of quantitative application of a corresponding theoretical kinetic model to either bulk or nanoscale systems.

Sato and co-workers previously observed photo-oxidation of Ge nanocrystals and showed a threshold for rapid oxidation just below $3 \mathrm{eV}$ which they attributed to direct excitation of carriers into the conduction band of the forming oxide followed by electron-assisted molecular oxygen dissociation [5]. While their data seem to indicate slow photo-oxidation under low intensity visible illumination, the focus of their work was on rapid photo-oxidation processes 
occurring at above-threshold energies and they did not propose a mechanism for photo-oxidation in the mid-visible range. In the present work, higher intensity illumination in this energy range is utilized in order to examine visible light photo-oxidation mechanisms.

Ge nanocrystals, with an average diameter of $6.3 \mathrm{~nm}$, were formed by ion implantation of ${ }^{74} \mathrm{Ge}^{+}$at $150 \mathrm{keV}$ to $3 \times 10^{16} \mathrm{~cm}^{-2}$ into $500 \mathrm{~nm}$ thick $\mathrm{SiO}_{2}$ layers on $\mathrm{Si}$ substrates followed by thermal annealing at $950{ }^{\circ} \mathrm{C}$ for $1 \mathrm{~h}$ under Ar. Exposed nanocrystals were obtained by selective etching of the silica matrix with 1:1 49\% $\mathrm{HF}: \mathrm{H}_{2} \mathrm{O}$, followed by immersion in methanol and drying under flowing $\mathrm{N}_{2}$. This selective etching process does not significantly alter the nanocrystal size distribution from the silica-embedded state [2]. Nanocrystals were exposed to air for times less than $30 \mathrm{~s}$ prior to photo-oxidation experiments; previous experiments revealed that hydrogen passivation suppresses oxidation for at least $2 \mathrm{~h}$ after etching [2].

Raman spectroscopy was performed in a macroscopic optical setup using the $515 \mathrm{~nm}$, $488 \mathrm{~nm}$, and $477 \mathrm{~nm}$ lines of an Ar laser and with a spot size of $2.6 \times 10^{-4} \mathrm{~cm}^{2}$ at the sample. The extent of oxidation was determined by monitoring the evolution of the Raman scattering intensity. At moderate and high intensities, photo-oxidation was measured in situ by continuous collection of Raman spectra at $1 \mathrm{~s}$ intervals. However, at low power illumination, the Raman scattering intensity was too low for in situ measurement. Therefore, reference spectra were collected for $1 \mathrm{~s}$ with moderate laser power, samples were then illuminated at reduced intensities for specific time intervals, and final spectra were collected for $1 \mathrm{~s}$ with moderate laser powers. For reference, some spectra were also collected under dry nitrogen.

Figure 1 shows the evolution of the integrated Raman intensity as a function of exposure time for various experimental configurations. All intensities are normalized to the integrated intensity of a $t=1 \mathrm{~s}$ exposure. Under nitrogen, there is no change of the intensity with 
illumination time. Furthermore, only a minimal decrease of the scattering intensity is observed during exposure of nanocrystals to ambient conditions and intermittent laser irradiation for measurement purposes only. Under continuous laser irradiation, however, a marked decrease of the scattering intensity is observed. Because the Raman intensity is proportional to the total scattering volume, the evolution of the scattering peak gives a direct measure of the extent of oxidation in real time and may be used to analyze the kinetics of the oxidation process. To ensure that laser heating of the sample is not responsible for the oxidation enhancement, the ratio of the anti-Stokes to Stokes scattering intensities was measured under $\mathrm{N}_{2}$ and yielded a maximum nanocrystal temperature of $306 \mathrm{~K}$. Therefore, the reduction of the Raman scattering intensity under room temperature illumination is assigned to photo-oxidation, rather than a thermal process.

The data points in Fig. 2 show the normalized integrated Raman intensity after $1250 \mathrm{~s}$ of illumination as a function of incident laser power and energy. The dependence of the oxidation rate on the laser intensity indicates that the oxidation process is not diffusion rate limited. For the case of the most highly-oxidized nanocrystals, the total remaining Ge volume is approximately 0.3 of the starting volume, which corresponds to formation of a $1 \mathrm{~nm}$ thick oxide shell surrounding the crystalline Ge core.

Within the electron-active photo-oxidation model, above bandgap light excites carriers inside Ge nanocrystals, thereby enhancing the tunneling probability of electrons through the growing oxide shell to molecular oxygen at the surface [4]. When an electron with energy greater than $1 \mathrm{eV}$ interacts with molecular oxygen, dissociation to a neutral oxygen atom and a negatively charged oxygen ion, both of which are more mobile than molecular oxygen, can occur 
[4]. The rate limiting step for photo-oxidation is the tunneling of electrons through the oxide for molecular oxygen dissociation.

We find, based on electron tunneling theory, that the time-dependent nanocrystal radius, $r$, is given by:

$$
\frac{d r}{d t}=-\frac{1}{3} r \eta I \alpha v \exp \left(\frac{-2 \sqrt{2 m^{*} U(r)}}{\hbar}\left(R_{0}-r\right)\right)
$$

where $I$ is the incident photon flux, $\alpha$ is the nanocrystal absorption coefficient, $v$ is the Ge volume consumed per oxidation event, $m^{*}$ is the electron effective mass $\left(=0.081 m_{0}\right.$ at the direct gap), $U(r)$ is the tunneling barrier, and $R_{0}(=3.15 \mathrm{~nm})$ is the average initial nanocrystal radius. Due to the small size of the nanocrystals, absorption is assumed to scale linearly with the volume such that the total number of absorbed photons per unit time is $I V \alpha$. The dimensionless term $\eta$ is an efficiency factor that is required to account for the rapid thermalization of photo-excited carriers to lower energy states, which decreases the tunneling probability.

UV photo-oxidation of both bulk Ge [6] and Ge nanowires yields primarily stoichiometric $\mathrm{GeO}_{2}$ [7], so the electronic properties of the $\mathrm{Ge} / \mathrm{GeO}_{2}$ interface are assumed for this analysis. The bulk valence band (VB) alignment was recently reported with $E_{V B}^{G e}-E_{V B}^{G e O_{2}}=2 e V$ [8]. However, the reduction of the Ge valence band energy with decreasing size, arising from quantum confinement, must be considered. The VB energy as a function of nanocrystal radius, calculated using the tight binding description, is [9]:

$$
E_{V B}^{G e}(r)=E_{V B}^{G E}(\infty)-\frac{15143.8}{4 r^{2}+12.93 r+2.546} \mathrm{meV}
$$

where $r$ is the nanocrystal radius in nanometers. Therefore, the size-dependent tunneling barrier may be written as: 


$$
U(r)=E_{g a p}^{G e O_{2}}-h v-\left(E_{V B}^{G e}(r)-E_{V B}^{G e O_{2}}\right)
$$

where $E_{V B}^{G e}(\infty)-E_{V B}^{G e O_{2}}=2 \mathrm{eV}, h v$ as the incident photon energy, and the bulk $\mathrm{GeO}_{2}$ bandgap, $E_{\text {gap }}^{\mathrm{GeO}_{2}}$, is equal to $5.6 \mathrm{eV}[10]$.

Though the bulk Ge absorption coefficient is clearly known over the energy range of interest, its value for Ge nanocrystals is not well established. Therefore, the parameter $C_{\lambda}=\eta \alpha(\lambda)$ is used for fitting the intensity dependence on the oxidation conversion for each wavelength. The solid lines in Fig. 2 show the results of fitting Eq. 3 to the experimental data for the variation of oxidation extent with laser intensity and energy using the parameters described above. Fitting yields the following values: $C_{476}=0.91 \mathrm{~cm}^{-1}, C_{488}=0.50 \mathrm{~cm}^{-1}$, and $C_{515}=0.22$ $\mathrm{cm}^{-1}$. Furthermore, the time-dependent volume conversion of nanocrystals obtained during two in situ Raman scattering experiments, one at $488 \mathrm{~nm}$ and one at $515 \mathrm{~nm}$, along with the corresponding simulated curves are shown in Fig. 1. There is excellent agreement between the shapes of these curves and the experimental data, which lends strong support for the proposed kinetic oxidation model.

Assuming a constant $\eta$ for the three excitation energies, the fitted values of $C_{\lambda}$ suggest an absorption coefficient that is four times larger at $476 \mathrm{~nm}$ than at $515 \mathrm{~nm}$, which is not the case for bulk Ge. The validity of strongly wavelength-dependent $C_{\lambda}$ fitting parameters was explored by monitoring the evolution of the Si substrate Raman signal during in situ photo-oxidation. Figure 3 shows the normalized Si Raman intensity as a function of time for in situ oxidation runs at three wavelengths. The vertical arrows indicate the times at which half of the Ge volume was consumed by photo-oxidation. Since germania is transparent at these wavelengths [10], the rise of the Si substrate Raman signal is a direct consequence of the reduction of Ge nanocrystal absorption. These data, along with some rather basic assumptions, can be used to obtain an 
approximate measurement of the average absorption coefficients at each wavelength during photo-oxidation. Our previous work indicated that approximately $70 \%$ of the total implanted Ge is retained on the surface after etching [2]. Therefore, assuming the remaining Ge forms a continuous film of the bulk Ge density and noting that both the excitation laser and the Raman scattered light both make single passes through the film, the following approximate absorption coefficients are calculated: $\alpha(476 \mathrm{~nm})=2.5 \times 10^{5} \mathrm{~cm}^{-1}, \quad \alpha(488 \mathrm{~nm})=1.6 \times 10^{5} \mathrm{~cm}^{-1}$, and $\alpha(515 \mathrm{~nm})=6 \times 10^{4} \mathrm{~cm}^{-1}$. The ratios of the calculated absorption coefficients are in excellent agreement with the ratios of the $C_{\lambda}$ fitting parameters required to describe the photo-oxidation results. These results further suggest that either the electronically confined direct transition, or an unidentified resonant transition, lies near the excitation energies. Further work will be required to specifically identify this electronic state.

Using the calculated absorption coefficients, the efficiency factor, $\eta$, is estimated to be $\sim 5 \times 10^{-6}$. This parameter is required to account for thermalization of photo-excited carriers and is expected to be small; thermalization increases the tunneling barrier and significantly reduces the fraction of electrons available to achieve oxygen dissociation at the surface.

In conclusion, photo-oxidation of air-exposed Ge nanocrystals was observed at room temperature under visible light illumination. Unlike in the well-known case of UV photooxidation, visible light does not possess enough energy to directly excite carriers into the conduction band of the forming oxide. Therefore, an electron-active oxidation mechanism, based on the tunneling of photo-excited carriers through the forming oxide skin followed by molecular oxygen dissociation and rapid atomic and/or ionic oxygen diffusion to the nanocrystal core, is proposed. Quantitative application of a kinetic model to time-resolved measurements of this process, including the laser energy and power dependencies, yields excellent agreement 
between experiment and theory using physical parameters. Since many synthesis routes lead to formation of exposed nanocrystals, it is of great importance to account for the possibility of photo-oxidation during subsequent optical measurements, even when using visible light.

\section{ACKNOWLEDGMENTS}

We would like to thank K.M. Yu for his input. D.C.C. and E.E.H. acknowledge support from the Miller Institute for Basic Research in Science. This work is supported in part by the Director, Office of Science, Office of Basic Energy Sciences, Division of Materials Science and Engineering, of the U.S. Department of Energy under contract No. DE-AC02-05CH11231 and in part by U.S. NSF Grant No. DMR-0405472.

\section{REFERENCES}

[1] B. E. Deal and A. S. Grove, J. Appl. Phys. 36, 3770 (1965).

[2] I. D. Sharp, Q. Xu, C. Y. Liao, D. O. Yi, J. W. Beeman, Z. Liliental-Weber, K. M. Yu, D. N. Zakharov, J. W. A. III, D. C. Chrzan and E. E. Haller, J. Appl. Phys. 97, 124316 (2005).

[3] N. F. Mott, Trans. Faraday Soc. 35, 1175 (1939).

[4] E. M. Young, Appl. Phys. A 47, 259 (1988).

[5] B. Sato, S. Nozaki and H. Morisaki, J. Appl. Phys. 81, 1518 (1997).

[6] V. Craciun, B. Hutton, D. E. Williams and I. W. Boyd, Electronics Lett. 34, 71 (1998).

[7] H. Adhikari, P. C. McIntyre, S. Sun, P. Pianetta and C. E. D. Chidsey, Appl. Phys. Lett. 87, 263109 (2005).

[8] V. V. Afanas'ev and A. Stesmans, Appl. Phys. Lett. 84, 2319 (2004).

[9] Y. M. Niquet, G. Allan, C. Delerue and M. Lannoo, Appl. Phys. Lett. 77, 1182 (2000).

[10] N. M. Ravindra, R. A. Weeks and D. L. Kinser, Phys. Rev. B 36, 6132 (1987). 


\section{FIGURE CAPTIONS}

Figure 1: Normalized integrated Raman intensity of air-exposed Ge nanocrystals as a function of time during in situ Raman spectroscopy under $515 \mathrm{~nm}$ illumination with a flux of $9.22 \times 10^{20}$ photons $\mathrm{cm}^{-2} \mathrm{~s}^{-1}(\square)$ and $488 \mathrm{~nm}$ illumination with a flux of $1.16 \times 10^{21}$ photons $\mathrm{cm}^{-2} \mathrm{~s}^{-1}(\circ)$. The solid lines show the predictions of the photo-oxidation theory presented in the text. Also shown is the Integrated Raman intensity from Ge nanocrystals as a function of time during continuous $488 \mathrm{~nm}$ laser illumination with a flux of $1.16 \times 10^{21}$ photons $\mathrm{cm}^{-2} \mathrm{~s}^{-1}$ under $\mathrm{N}_{2}(\Delta)$ and for intermittent laser exposure under air (घ). All data points are normalized to the intensity of the $t=$ $1 \mathrm{~s}$ laser exposure of each data series.

Figure 2: Normalized integrated Raman intensity from Ge nanocrystals as a function of energy and photon flux after $1250 \mathrm{~s}$ of illumination under air (data points). The solid lines show the calculated values according to the theory presented in the text.

Figure 3: Si substrate Raman intensities, normalized to the intensity at $t=1 \mathrm{~s}$, as a function of time during photo-oxidation of Ge nanocrystals under $476 \mathrm{~nm}$ illumination with $4.81 \times 10^{20}$ photons $\mathrm{cm}^{-2} \mathrm{~s}^{-1}(\square), 488 \mathrm{~nm}$ illumination with $2.00 \times 10^{21}$ photons $\mathrm{cm}^{-2} \mathrm{~s}^{-1}(\circ)$, and $515 \mathrm{~nm}$

illumination with $1.82 \times 10^{21}$ photons $\mathrm{cm}^{-2} \mathrm{~s}^{-1}(\Delta)$. The intensity increase is due to reduced photon absorption of Ge on the surface. Arrows indicate the times at which one half of the initial Ge volume is consumed by photo-oxidation for each data series. 


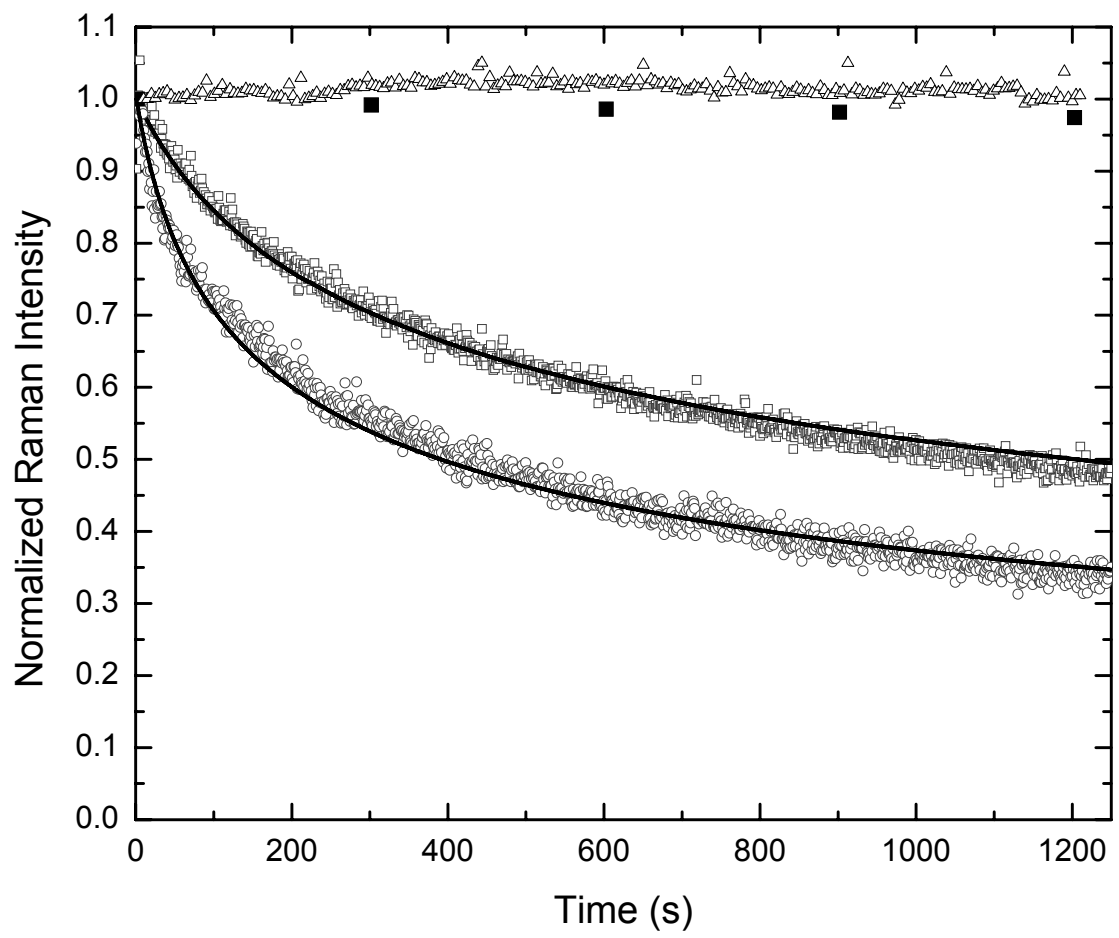

Figure 1 


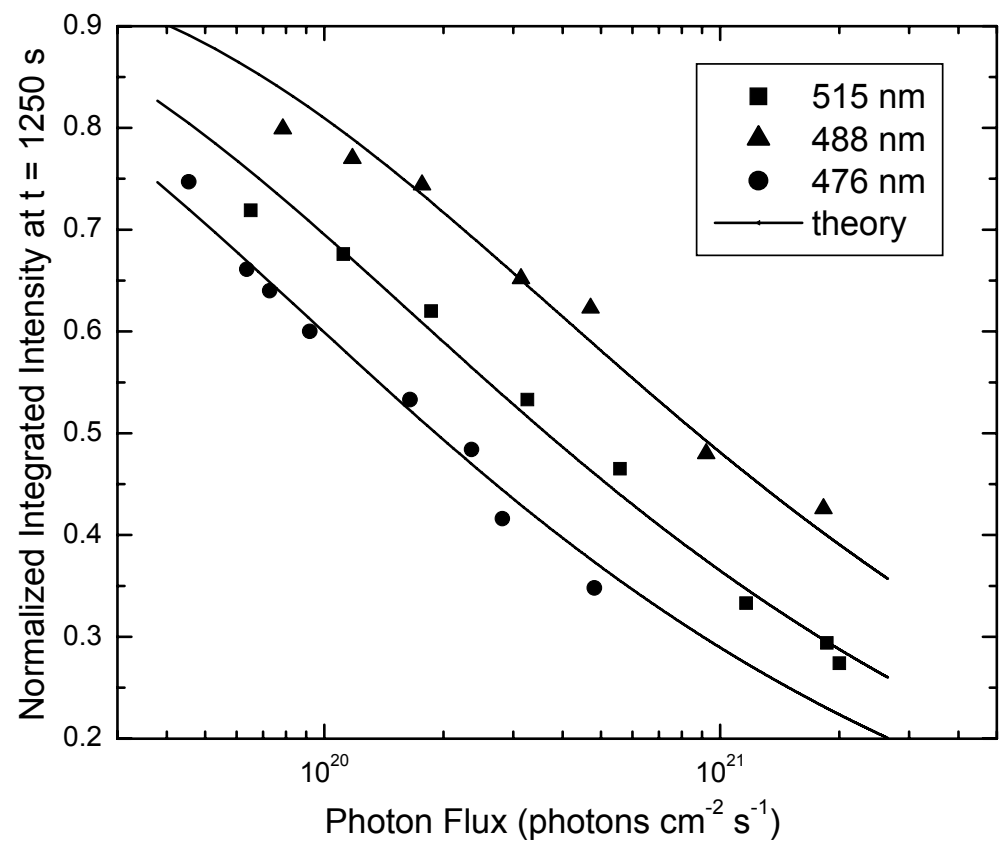

Figure 2 


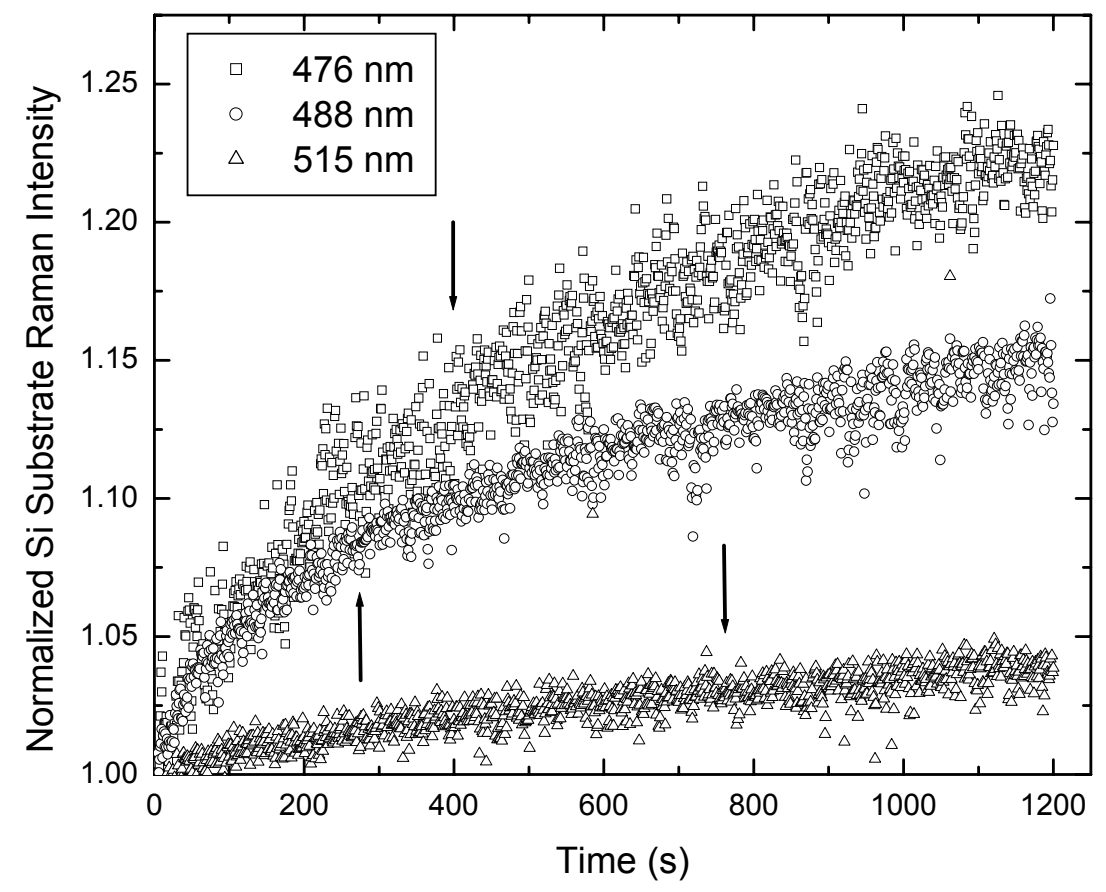

Figure 3 